Proceedings of the 11th Polish-Japanese Joint Seminar on Micro and Nano Analysis, Gniew, September 11-14, 2016

\title{
Application of Graphics Processing Unit for In-Line Electron Holography
}

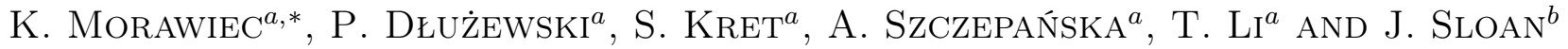 \\ ${ }^{a}$ Institute of Physics, Polish Academy of Sciences, Aleja Lotnikow 32/46, PL-02668 Warsaw, Poland \\ ${ }^{b}$ Department of Physics, University of Warwick, Coventry CV4 7AL, United Kingdom
}

\begin{abstract}
In the present work, software for exit electron wave reconstruction based on the iterative approach was implemented and a new method for drift-correction of the focal series was proposed.

DOI: 10.12693/APhysPolA.131.1353

PACS/topics: 68.37.Lp, 68.37.Og, 68.55.Nq, 42.30.Rx, 61.05.jp, 07.05.Pj, 42.30.-d, 42.30.Wb
\end{abstract}

\section{Introduction}

Due to the electron-optic aberrations present in transmission electron microscope (TEM) a single image does not allow for direct interpretation of the investigated structure. For instance, in the case of high resolution TEM imaging, a small change in the defocus value can already cause a drastic change in the observed crystal pattern (see Fig. 1a and b). This change could wrongly suggest the positions of atomic columns in the sample. In order to observe the exact specimen structure one could move the specimen to the focal plane of an objective lens, but then the amplitude contrast on the screen would be very weak. Fortunately it is possible to use a series of differently defocused TEM images (i.e. a focal series) to restore the full available information about both amplitude and phase of the exit electron wave. This procedure is known as exit wave reconstruction (EWR, i.e. in-line electron holography) and, in theory, when connected with determination and correction of aberrations, it allows for expanding the image resolution up to the information limit of the microscope. In practice it may also provide a significant increase in image contrast due to the restoration of phase which is not directly available during the conventional TEM experiment. This is important in the case of weak phase objects, for which the phase of transmitted electron wave is affected much more than its amplitude, and it strongly depends on the electrostatic projected potential of the sample [1].

There are already many in-line holography algorithms developed each one of them implementing a little bit different approach to the problem. Among them the most important methods are the full-resolution in-line holography (FRIH), developed by Koch [2], and focal and also the tilt series reconstruction (FTSR), developed by Meyer et al. [3, 4]. However, in the current work the iterative approach known as the iterative wave function reconstruction (IWFR) was implemented based on the ideas of Allen et al. [5] and Ishizuka [6].

*corresponding author; e-mail: morawk@ifpan.edu.pl
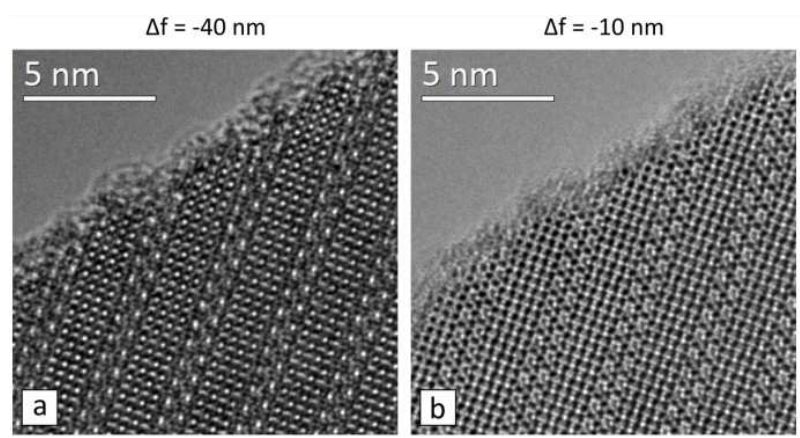

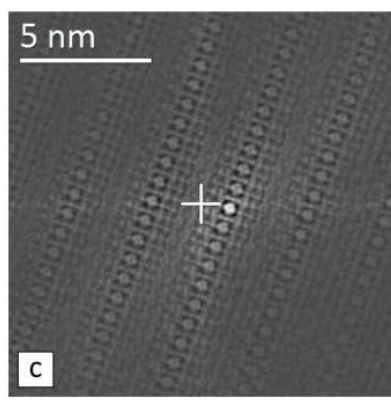

MCF as-is

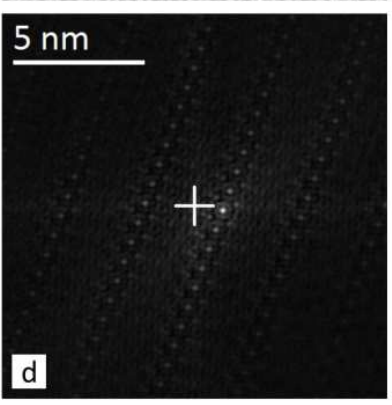

MCF with propagation
Fig. 1. (a,b) experimental HRTEM images coming from the same focal series, showing the same area of a tungsten bronze specimen for defocus $\Delta f=-40 \mathrm{~nm}$ (a) and $\Delta f=-10 \mathrm{~nm}$ (b). (c) MCF image of two original HRTEM images, (d) MCF image obtained after "propagation" of the first TEM image towards the plane of the second TEM image through the distance of $\Delta z \approx 30 \mathrm{~nm}$. The displacement of MCF peak position with respect to the center of MCF image (indicated by the white cross) corresponds to the in-plane shift between TEM images. It should be noted that the contrast limits are the same for both MCF images.

If the microscope is equipped with the aberration corrector, then in the first approximation the consideration of higher order aberrations can be neglected and the defocus becomes a crucial aberration in exit wave reconstruction and this fact allows for utilization of a simple defocus dependent Fresnel propagator [5].

Due to the mechanical drift which is often present during the acquisition of focal series, it is necessary to align 
all the images to the common origin before running the exit wave reconstruction procedure. Image drift alignment is a crucial step for effective restoration of exit electron wave and it can be automated with the use of crosscorrelation function ( $\mathrm{CCF}$ ) analysis. However, due to the fact that the simple cross-correlation approach is not suitable for all types of specimens and focal series, some modifications to this method have to be made. In particular, the standard CCF was replaced with its mutual variant, called the mutual-correlation function (MCF). It has been found that the MCF gives the correlation peak which is much sharper and more localized, thus allowing for easier determination of shift between two images [3]. The expressions for both - CCF and MCF - are as follows:

$$
\begin{aligned}
& \operatorname{CCF}(x, y)=F T^{-1}\left(C_{1}^{*} C_{2}\right), \\
& \operatorname{MCF}(x, y)=F T^{-1}\left(\sqrt{\left|C_{1}^{*} C_{2}\right|}\right),
\end{aligned}
$$

where $C(h, k)$ is a Fourier transform $(F T)$ of an image contrast $c(x, y)$, the asterisk indicates complex conjugation and where $F T^{-1}$ stands for the inverse Fourier transform. As one can see, both definitions are very similar and the only difference between them is the square root which appears in the expression for the MCF.

In general, the mechanical drift causes the specimen to move in all $x, y$ and $z$ directions. Additionally, there is also the intentional change of defocus which manifests itself in the reduction of the Fresnel fringes, observed on the edges of the specimen, when coming towards zero defocus. Consider the two images shown in Fig. 1a and $b$ which were recorded for a thin crystal of a tungsten bronze $\left(\mathrm{K}_{x} \mathrm{WO}_{3}\right)$. This data was previously used for exit wave reconstruction with other algorithms by one of the co-authors and the results were published [7]. The difference in defocus between two presented images was caused by changing the current of the objective lens. In order to align images as accurately as possible the following modifications were introduced to the alignment procedure as well.

First of all the fact that the correlated images come from different image planes and correspond to different defocus values had to be addressed. More specifically, the goal was to minimize the effect of defocus on the change of contrast between images [3]. For this purpose it was proposed to "propagate" the amplitude (i.e. square root of intensity) of one image towards the plane of another in order to correlate their intensities at the same plane (i.e. the same $z$-distance from the focal plane). This solution makes the images become more comparable and allows for the $(x, y)$-shift to be determined more accurately. In the real space the electron wave function propagated across a $\Delta z$ distance can be written as follows:

$$
\psi_{2}(x, y)=\psi_{1}(x, y) \otimes t(x, y, \Delta z) .
$$

However, it is more convenient to consider the electron wave propagation in the reciprocal space, because then the convolution $(\otimes)$ turns into simple multiplication ac- cordingly to the following equation:

$$
\begin{gathered}
\Psi_{2}(h, k)=\Psi_{1}(h, k) \cdot T(h, k, \Delta z)= \\
\Psi_{1}(h, k) \cdot \exp \left(-\mathrm{i} \pi \lambda \Delta z\left(h^{2}+k^{2}\right)\right),
\end{gathered}
$$

where $\Psi(h, k)=F T(\psi(x, y)), \quad T(h, k, \Delta z)=$ $F T(t(x, y, \Delta z))$ is the Fresnel propagator in vacuum, $\lambda$ is the electron wavelength and $(h, k)$ are the coordinates of a vector $\boldsymbol{g}$ in reciprocal space. Normally the process of propagation requires knowledge of initial phase values, which have a significant effect on how the image looks like after the propagation. However, for the purpose of simplicity of the alignment method presented in this work and due to the fact that the phase values of registered images are yet to be restored, the phase is neglected from calculations of the propagated wave (i.e. it is assumed to be zero). One should keep in mind that the above expression, utilizing the Fresnel propagator in a vacuum, has its limitations and is just a simplified approximation of how the wave propagation occurs in a real TEM optical system.

Figure 1 presents the outcomes of two approaches to the MCF procedure, i.e. an MCF applied to a pair of unmodified TEM images (Fig. 1c) and an MCF which additionally utilizes the propagation between image planes (Fig. 1d). Calculations were performed on the basis of two HRTEM images that are slightly shifted and defocused with respect to each other, see Fig. 1a and b. In both presented MCF images the distance between the center of an image and the position of its maximum intensity corresponds to the $(x, y)$-shift between two considered TEM images. As one can see there is a significant improvement in localization of the correlation peak when using the MCF with propagation of amplitude.

In this paper the following solutions were proposed in order to improve the drift-correction procedure even more. First modification was to split the images into square fragments and correlate respective fragments separately. In this way only the fragments which contain characteristic sample features can be included in calculations of average shift between two images. Moreover, those fragments, which have almost uniform contrast (e.g. thin amorphous film) and could affect the alignment procedure negatively, can be neglected from the calculations making the result much more reliable.

A second new feature introduced in this work for the purpose of image drift alignment was to iteratively change the defocus between correlated images in order to obtain the sharpest correlation peak and consequently an actual $\Delta z$ value which depends on both a specimen drift in the $z$ direction and the change of defocus. This solution not only improves the drift correction, but also allows the determination of the relative defocus values which are very important for the correct restoration of the exit electron wave. In this work the experimental image with the lowest contrast and the absence of Fresnel fringes was assumed to be in-focus (i.e. have zero defocus). With this assumption and the knowledge of 
defocus differences between all successive pairs of images, it was possible to achieve a good estimate of the absolute defocus values.

\section{Experiment}

For the purpose of this work, software for solving the exit wave reconstruction problem was implemented using the Python language and also the CUDA environment for GPU (graphics processing unit) programming. Using a graphics processor and its specific architecture allowed for improving the computation speed by parallelization of many calculations. All computations were conducted on NVIDIA Tesla K20c GPU.

The exit wave function was refined for two kinds of specimens, for which different imaging conditions were used. The first specimen consisted of amorphous acrylicpolymer nanoparticles, which were supposed to have the core-shell structure. The imaging was performed in the conventional TEM mode. The focal series consisted of twenty images on both over- and under-focus sides of the specimen plane. The defocus step between successive images was set to be constant and equal to $50 \mathrm{~nm}$.

The second specimen consisted of the Mn-rich nanoprecipitations embedded in a GaAs matrix. In this case, the focal series was acquired in the Lorentz imaging mode (i.e. with the objective lens switched off and Lorentz mini-lens switched on) to accompany off-axis holography measurements conducted to obtain the distribution of magnetic fields in the sample. The focal series acquired for this specimen consisted of twenty images and the defocus step was set to $2 \mu \mathrm{m}$.

In both cases, image sizes of $1024 \times 1024$ pixels were registered on a CCD camera and the exposure time for a single acquisition was $2 \mathrm{~s}$. All measurements were conducted on the Cs-corrected Titan $^{3}$ 80-300 instrument.

\section{Results and discussion}

During the focal series measurements a mechanical drift of the specimen was always present. In order to automatically align the acquired images the iterative MCF with fragmentary correlation (as described in the introduction) was applied for each pair of successive images. As expected the iterative-fragmentary MCF method (IFMCF) turned out to be very efficient in the case of both datasets allowing not only for a proper drift-correction, but also for a reliable estimation of absolute defocus values.

Figure 2 shows the results of exit wave reconstruction algorithm applied to the focal datasets of two investigated specimens: acrylic-polymer particles (Fig. 2a-c) and MnAs precipitations embedded in GaAs matrix (Fig. 2df). In Fig. 2a and d one can see the experimental TEM images acquired near zero-defocus. In both cases amplitude and phase images restored after 10 iterations of IWFR procedure are presented, see Fig. 2b,c and 2e,f.

In the case of acrylic-polymer nanoparticles, the calculated amplitude image shows a significant increase in contrast on the edge of the particles, indicating that these are, as expected, the core-shell structures.

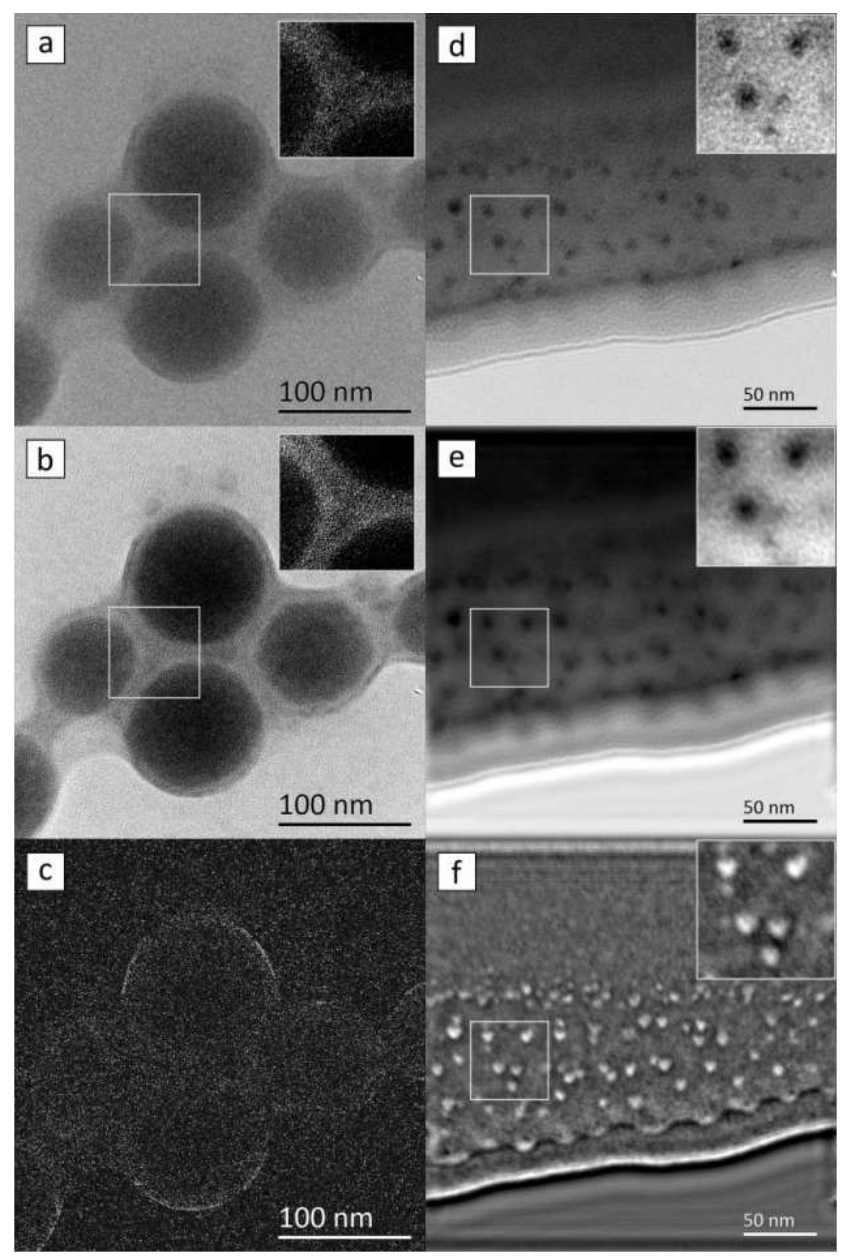

Fig. 2. Experimental TEM images (a,d) and the results of EWR consisting of restored amplitude images $(\mathrm{b}, \mathrm{e})$ and restored phase images $(\mathrm{c}, \mathrm{f})$ for two kinds of specimens. In the case of acrylic-polymer particles coreshell structure can be distinguished in the restored amplitude image. Phase image of the second specimen shows a significant increase in the contrast between Mnrich precipitations and GaAs matrix when compared to the experimental image. Phase image shown in Fig. 1c was digitally enhanced by a factor of 50 in order to draw out some of the structure details, i.e. the modulo operation has been applied to the phase image.

In the experimental image of the second specimen the Mn-rich nanoprecipitations barely stand out from the GaAs matrix, while in the restored phase image they are clearly distinguishable and their size can be defined more precisely.

It is worth mentioning that the in-line holography could also be a complementary or even competitive method for off-axis holography in terms of determination of distribution of magnetic field in the sample. This however would require measurements in the Lorentz mode of TEM so that the internal magnetic field of the sample is not affected by the strong magnetic field of an objective lens. Moreover, acquisition of an additional focal series 
showing the same area in the upside-down position of the specimen would be necessary. Phase images restored from these two focal series could then be used to subtract one phase map from the other in order to get the "magnetic phase" which is related to the magnetic field in the specimen. Similarly, the sum of reconstructed phase images would be related to the distribution of electrostatic potential of the sample. It is known that while off-axis holography performs better in restoring low spatial frequencies, in-line holography is more effective in terms of resolving higher spatial frequencies. This fact is used in a technique called hybrid electron holography [8].

\section{Conclusions}

Exit wave reconstruction can be a very useful supplementary method for high-resolution TEM imaging in terms of characterization of the crystal structure of the specimen. It allows for the restoration of information which is not directly available in conventional TEM, in particular the phase of exit electron wave. Thereby it provides the possibility to observe a real specimen structure, as it would be in the focal plane of the objective lens, but with much better contrast and resolution coming from the fact that a full range of spatial frequencies is included in calculations.

EWR can as well be applied to the "phase objects", such as the core-shell amorphous nanoparticles, for which the density change between two phases (i.e. the core and the shell) is very slight, causing the contrast between them to be at the very low level. Fortunately, EWR can be used to restore indirectly available information about the specimen in order to enhance the contrast between two phases.

In the case of the drift-correction procedure presented in this work, there is still room for improvement. One of the possible solutions is to combine the process of alignment with the process of reconstruction and thus create a self-consistent algorithm. In this way the successive approximations of the restored phase could be used to align images even more precisely and allow for better refinement of exit electron wave function.

\section{Acknowledgments}

This research was co-financed by the Polish National Science Center (Grant No. UMO2013/11/B/ST3/04244) and by the European Regional Development Fund within the Innovative Economy Operational Programme 2007-2013 (Grant No. POIG.02.01-00-14-032/08). We are also grateful for additional support from the EAgLE international project (FP7-REGPOT-2013-1, Project No. 316014) co-financed by Polish Ministry of Science and Higher Education, Grant Agreement 2819/7.PR/2013/2.

Samples used for registration of TEM focal series utilized in this work were provided by courtesy of Dr. J. Sadowski (Institute of Physics, PAS) and Prof. Dr. J. Kozakiewicz (Industrial Chemistry Research Institute, PAS). Focal series of tungsten bronze $\left(\mathrm{K}_{x} \mathrm{WO}_{3}\right)$ was provided by one co-author (J.S.).

\section{References}

[1] M.A. Dyson, Ph.D. Thesis, University of Warwick, Coventry 2014.

[2] C.T. Koch, Micron 63, 69 (2014).

[3] R.R. Meyer, A.I. Kirkland, W.O. Saxton, Ultramicroscopy 92, 89 (2002).

[4] R.R. Meyer, A.I. Kirkland, W.O. Saxton, Ultramicroscopy 99, 115 (2004).

[5] L.J. Allen, W. McBride, N.L. O'Leary, M.P. Oxley, Ultramicroscopy 100, 91 (2004).

[6] K. Ishizuka, Microscopy 62, S109 (2013).

[7] A.I. Kirkland, J. Sloan, S. Haigh, Ultramicroscopy 107, 501 (2007).

[8] C. Ozsoy-Keskinbora, C.B. Boothroyd, R.E. DuninBorkowski, P.A. van Aken, C.T. Koch, Sci. Rep. 4, 7020 (2014). 